\title{
Nomogram construction for predicting survival of patients with non-small cell lung cancer with malignant pleural or pericardial effusion based on SEER analysis of 10,268 patients
}

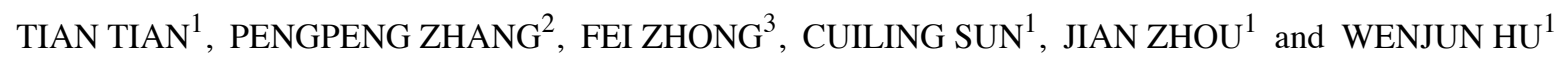 \\ ${ }^{1}$ Department of Medical Oncology, Fuyang People's Hospital; ${ }^{2}$ Medical Imaging Center, Fuyang Second People's Hospital; \\ ${ }^{3}$ Department of Medical Oncology, Affiliated Fuyang Hospital of Anhui Medical University, Fuyang, Anhui 236000, P.R. China
}

Received May 13, 2019; Accepted October 29, 2019

DOI: $10.3892 / \mathrm{ol} .2019 .11112$

\begin{abstract}
Determining the accurate outcome of patients with non-small cell lung cancer (NSCLC) and malignant pleural effusion (MPE) or malignant pleural pericardial effusion (MPCE) at the initial diagnosis remains a challenge. The aim of the present study was to develop an effective nomogram for individualized estimation of overall survival in these patients. Patients diagnosed between January 2010 and December 2015 were selected from the Surveillance, Epidemiology, and End Results (SEER) database. Age, race, sex, grade, histology, laterality, stage and status of MPE or MPCE at initial diagnosis were included as covariates. Several survival models were created and the performance of each was evaluated. The most effective model was then validated by internal bootstrap resampling and by using an independent external cohort. A nomogram was created based on this survival model and the predictive accuracy of the nomogram was evaluated by calibration plots. Data from 10,268 patients with lung cancer with MPE or MPCE at initial diagnosis were collected. The multivariate analysis with a lognormal model suggested that age, race, sex, histology, stage and status of MPE or MPCE at initial diagnosis were significant independent factors to predict survival. A nomogram was constructed based on the lognormal survival model, which showed the best performance. The concordance index of the survival model in the SEER cohort was 0.736 . Both internal and external validation showed an acceptable level of agreement between the nomogram-predicted survival probability and actual survival. The nomogram of the present study based on a large cohort from the SEER database may improve prognostic prediction
\end{abstract}

Correspondence to: Professor Wenjun Hu or Dr Tian Tian, Department of Medical Oncology, Fuyang People's Hospital, 501 Sanqing Road, Fuyang, Anhui 236000, P.R. China

E-mail: 302320883@qq.com

E-mail: tiannt@foxmail.com

Key words: non-small cell lung cancer, malignant pleural effusion, malignant pericardial effusion, prognosis of patients with NSCLC with MPE or MPCE at initial diagnosis, and allow physicians to make appropriate decisions for disease management of their patients.

\section{Introduction}

Lung cancer is a significant global health problem, with an estimated total of 228,150 new cases and 142,670 deaths in the United States in 2019 (1). Lung cancer is the leading cause of cancer-related death worldwide (2). Non-small cell lung cancer (NSCLC) and small cell lung cancer are the two major histological categories of lung cancer. Patients with NSCLC occasionally present with malignant pleural effusion (MPE) or pericardial effusion (MPCE) at the initial diagnosis (3) and these patients are classified as being in the M1 stage according to the 7th edition of the American Joint Committee on Cancer (AJCC) tumor-node-metastasis (TNM) staging system (4). The median survival time of these patients with the same stage can differ from 3 months to 1 year (5). Determining the accurate outcome for specific patients remains a challenge.

There are several published studies on the survival prediction of patients with MPE and MPCE (6-8), most of which are dependent on biomarker concentrations in the effusions. However, to the best of our knowledge, a survival model specifically describing the prognosis of patients with MPE or MPCE with different demographic and clinicopathological characteristics is not available. Predicted survival information from a nomogram may assist patients and physicians in making appropriate decisions with regards to management.

The aim of the present study was to construct a survival model capable of predicting prognosis of patients with stage IV NSCLC with MPE or MPCE at initial diagnosis, using the data in the Surveillance, Epidemiology, and End Results (SEER) database and to establish a nomogram to illustrate the association between the prognostic factors and overall survival (OS).

\section{Patients and methods}

Study population. The present study was approved by The Ethics Committee of Fuyang People's Hospital. Permission was obtained from the Surveillance, Epidemiology and End Results (SEER) Program to access the SEER research data 
files (reference no. 16924-Nov2017). Informed consent was obtained from each patient in the validation dataset. However, consent in the SEER training cohort was waived considering the anonymous, observational, registry-based and publicly available nature of the data. Patient data on individuals was not reported.

The patient cohort data for the training dataset were obtained from the SEER Program (seer.cancer.gov) SEER*Stat Database. Initial patient selection was performed by specifying the site recode as 'Lung and Bronchus'. Patients with MPE or MPCE at initial diagnosis who were diagnosed between January 2010 and December 2015 were included for further study by selecting 'CS Mets at DX' with codes 15-18, 20-21, 32, 42 and 52. The codes were defined as follows: 15 , malignant pleural effusion, ipsilateral or same lung; 16, malignant pleural effusion, contralateral or other lung; 17, malignant pleural effusion, ipsilateral and contralateral lungs; 18 , malignant pleural effusion, unknown if ipsilateral or contralateral lung; 20, malignant pericardial effusion; 21 , malignant pericardial effusion plus contralateral or bilateral pleural effusion; 32 , distant lymph nodes plus pleural or pericardial effusion; 42, distant metastasis plus extension to contralateral lung; and 52 , distant metastasis plus distant lymph nodes plus pleural or pericardial effusion. The exclusion criteria were as follows: i) Status of MPE or MPCE was unknown at initial diagnosis; ii) missing or incomplete information regarding race, stage, grade, histology, primary site or laterality; and iii) death certificate only or autopsy only cases in the SEER database. A total of 10,268 patients from the SEER database were included, comprising 5,827 (56.7\%) men and 4441 (43.3\%) women. The median age at diagnosis in the training dataset was 70 years (age range, 21-86 years).

The independent external validation dataset consisted of patients with NSCLC with MPE or MPCE at initial diagnosis between January 2013 and January 2018 who were hospitalized at three institutions in China (Fuyang People's Hospital, Fuyang Second People's Hospital and Affiliated Fuyang Hospital of Anhui Medical University; all Fuyang, China). Patient data, including survival time, age, race, sex, grade, histology, laterality, stage, physical status, LDH levels in effusion, neutrophil-to-lymphocyte ratio and status of MPE or MPCE at initial diagnosis, were collected to validate the nomogram and to compare with previous predictive model (7). Patients were followed-up every 2 weeks and the observations for this dataset were censored on January 1st, 2019. Patients with missing values for the above variables were excluded. In the validation dataset, 169 patients $(54.2 \%)$ were men, and 143 patients $(45.8 \%)$ were women. The median age at diagnosis in the external validation dataset was 72 years (age range, 25-87 years).

Statistical analysis. Age, race, sex, grade, histology, laterality, AJCC 7th edition TNM stage and status of MPE or MPCE at initial diagnosis were included from the SEER database as the factors in the training cohort. Using this cohort, several multivariate regression models were built, including parametric models and semiparametric models. The performance of the models was compared using the Akaike information criteria (AIC), where the lowest AIC value suggested the best predictive performance. This method has been reported in previous studies on the construction and evaluation of survival models $(9,10)$. The concordance index (C-index) was calculated to evaluate the discriminatory ability of the survival model. A calibration curve was created to show the difference between the predicted and actual survival rate, and data represented the means \pm standard error of the mean. A nomogram was created based on the survival prediction model with the lowest AIC. Internal validation was performed using bootstrap resampling, while external validation was performed using an independent cohort. The size of the external validation population was calculated using Vergouwe's method (11). To determine the discrimination ability of the nomogram, the total scores from the nomogram for each patient in the training and validation datasets were calculated. The patients were then divided into four prognosis groups according to the quartiles of the predicted survival of the training dataset, which were regarded as cutoffs for both the training and validation cohorts. Kaplan-Meier survival curves were constructed for certain TNM categories (M1a and M1b) and all categories in both datasets with the above cutoffs, and the log-rank test was applied for each category. To assess the usefulness of the nomogram and the LENT (pleural fluid lactate dehydrogenase, Eastern Cooperative Oncology Group performance status, neutrophil to lymphocyte ratio and tumor type) scoring system (7), receiver operating characteristic (ROC) curves for 3-, 6-, 9- and 12-month survival rates were calculated, and the areas under the curves (AUCs) were also calculated and compared. ROC curves were compared using the DeLong method (12). Statistical analyses were formed using R (version 3.5.2; The R Foundation), MedCalc software (version 11.4; MedCalc Software bvba) and SPSS software (version 25.0; IBM Corp.)

\section{Results}

Patient characteristics. A total of 10,268 patients from the SEER database were included in the final population for the survival analysis. Of these, 7,562 patients (73.6\%) had adenocarcinomas and 2,706 patients (26.4\%) had other histological subtypes, such as large cell carcinomas or squamous cell carcinomas. A total of 5,635 patients (54.9\%) exhibited right laterality, 4,075 patients (39.7\%) exhibited left laterality and 558 patients (5.4\%) had bilateral lesions. This SEER cohort was used as the training dataset. The independent external validation dataset consisted of 312 patients, of whom 266 patients (85.3\%) had adenocarcinomas, and 46 patients (14.7\%) had other histological types. A total of 175 patients (56.1\%) exhibited right primary site laterality, 110 patients (35.2\%) exhibited left primary site laterality and 27 patients $(8.7 \%)$ exhibited bilateral lesion of the primary site. The demographic and clinicopathological characteristics of both cohorts are listed in Table I.

OS of the training and validation cohorts. The median survival time of the training cohort was 7 months [95\% confidence interval (CI), 6.8-7.2 months]. The 3-, 6-, 9- and 12-month survival rates of the training cohort were 71.0, 52.4, 38.5 and $28.3 \%$, respectively. The median survival time of the validation cohort was 12.0 months (95\% CI, 11.0-13.0 months). The 3-, 6-, 9- and 12-month survival rates of the validation cohort were $85.2,72.6,59.4$ and $44.5 \%$, respectively (data not shown). 
Table I. Demographic and clinicopathological characteristics of the training cohort and validation cohort.

A, Training cohort, $n=10268$

Clinicopathological characteristics

Sex

Male

Female

Age at diagnosis, years

$\leq 70$

$>70$

Race

American Indian/Alaska Native

Asian or Pacific Islander

Black

White

Primary site

Main bronchus

Upper lobe

Middle lobe

Lower lobe

Overlapping lesion

Lung, NOS

Histology

Large cell carcinoma

Adenocarcinoma

Squamous cell carcinoma

Grade

I

II

III

IV

Unknown

Laterality

Right

Left

Bilateral

T stage

T0

T1

T2

T3

T4

Tx

$\mathrm{N}$ stage

NO

N1

N2

N3

$\mathrm{Nx}$

M stage

M1a

M1b
Number of patients (\%)

$5,827(56.7)$

4,441 (43.3)

$5,171(50.4)$

5,097 (49.6)

$43(0.4)$

881 (8.6)

$1,472(14.3)$

7,872 (76.7)

635 (6.2)

4,228 (41.2)

381 (3.7)

$2,500(24.3)$

$156(1.5)$

2,368 (23.1)

$246(2.4)$

$7,562(73.6)$

2,460 (24)

219 (2.1)

1,221 (11.9)

2,211 (21.5)

$95(0.9)$

$6,522(63.5)$

$5,635(54.9)$

4,075 (39.7)

558 (5.4)

111 (1.1)

495 (4.8)

$2,522(24.6)$

2,430 (23.7)

3,083 (30)

1,627 (15.8)

2,174 (21.2)

550 (5.4)

4,571 (44.5)

2,167 (21.1)

$806(7.8)$

4,611 (44.9)

$5,657(55.1)$
Median OS, months (CI)

\author{
$6(5.76-6.24)$ \\ $8(7.67-8.33)$ \\ 9 (8.68-9.32) \\ $5(4.79-5.21)$ \\ $5(1.85-8.15)$ \\ $12(11.12-12.88)$ \\ 7 (6.42-7.58) \\ 7 (6.80-7.20)
}

$5(4.38-5.62)$

7 (6.69-7.31)

$8(7.04-8.96)$

$8(7.60-8.40)$

$5(3.72-6.28)$

$6(5.62-6.38)$

$4(3.21-4.78)$

$7(6.77-7.23)$

$6(5.65-6.35)$

$10(8.22-11.79)$

8 (7.35-8.66)

7 (6.59-7.41)

$4(2.57-5.43)$

7 (6.76-7.24)

7 (6.74-7.26)

7 (6.69-7.31)

$6(5.26-6.74)$

8 (6.21-9.80)

10 (8.91-11.09)

8 (7.56-8.44)

7 (6.56-7.44)

$6(5.69-6.32)$

6 (5.53-6.48)

9 (8.52-9.48)

8 (7.03-8.97)

$6(5.75-6.25)$

7 (6.60-7.40)

5 (4.44-5.56)

$10(9.65-10.35)$

5 (4.79-5.21) 
Table I. Continued.

\begin{tabular}{lcc}
\hline Clinicopathological characteristics & Number of patients $(\%)$ & Median OS, months (CI) \\
\hline Effusions at diagnosis & & $10(9.57-10.43)$ \\
MPE, Ipsilateral & $3,028(29.5)$ & $10(8.31-11.69)$ \\
MPE, Contralateral & $145(1.4)$ & $8(7.12-8.88)$ \\
MPE, Bilateral & $524(5.1)$ & $8(7.05-8.95)$ \\
MPE, unknown & $342(3.3)$ & $10(8.98-11.02)$ \\
MPCE & $366(3.6)$ & $8(6.75-9.25)$ \\
MPCE + MPE & $206(2.0)$ & $9(8.00-10.00)$ \\
DLN + MPE or MPCE & $336(3.3)$ & $5(4.78-5.22)$ \\
DM + MPE or MPCE & $4,329(42.1)$ & $5(4.54-5.46)$ \\
DM + DLN + MPE or MPCE & $992(9.7)$ & \\
\hline
\end{tabular}

B, Validation set, $\mathrm{n}=312$

Clinicopathological characteristics

Sex

Male

Female

Age at diagnosis, years

$\leq 70$

$>70$

Race

American Indian/Alaska Native

Asian or Pacific Islander

Black

White

Primary site

Main bronchus

Upper lobe

Middle lobe

Lower lobe

Overlapping lesion

Lung, NOS

Histology

Large cell carcinoma

Adenocarcinoma

Squamous cell carcinoma

Grade

I

II

III

IV

Unknown

Laterality

Right

Left

Bilateral

T stage

T0

$\mathrm{T} 1$

T2
Number of patients (\%)

Median OS, months (CI)

$169(54.2)$

$143(45.8)$

$11(9.54-12.46)$

$12(10.27-13.73)$

$140(44.9)$

$14(12.23-15.77)$

$172(55.1)$

9 (7.37-10.64)

NA

312 (100)

NA

NA

$13(4.2)$

$119(38.1)$

$11(3.5)$

$69(22.1)$

7 (2.2)

93 (29.8)

5 (1.6)

$266(85.3)$

$41(13.1)$

$219(70.2)$

4 (1.3)

$41(13.1)$

48 (15.4)

NA

$175(56.1)$

$110(35.2)$

27 (8.7)

5 (1.6)

$10(3.2)$

67 (21.5)
NA

12 (11.04-12.96)

NA

NA

9 (2.64-15.36)

13 (11.86-14.14)

12 (4.72-19.28)

12 (9.73-14.27)

4 (1.43-6.57)

$9(6.38-11.62)$

$2(0.00-4.15)$

12 (10.83-13.17)

9 (6.44-11.56)

11 (9.56-12.45)

21 (NA)

13 (11.93-14.07)

13 (9.53-16.47)

NA

12 (10.95-13.05)

$12(9.55-14.45)$

11 (5.33-16.68)

NA

25 (0.84-49.17)

14 (10.99-17.01) 
Table I. Continued.

\begin{tabular}{|c|c|c|}
\hline Clinicopathological characteristics & Number of patients (\%) & Median OS, months (CI) \\
\hline $\mathrm{T} 3$ & $67(21.5)$ & $13(10.16-15.84)$ \\
\hline $\mathrm{T} 4$ & $93(29.8)$ & $11(8.03-13.97)$ \\
\hline $\mathrm{Tx}$ & $70(22.4)$ & $9(6.77-11.23)$ \\
\hline \multicolumn{3}{|l|}{$\mathrm{N}$ stage } \\
\hline No & $52(16.7)$ & $12(9.53-14.47)$ \\
\hline N1 & $15(4.8)$ & $14(10.77-17.23)$ \\
\hline $\mathrm{N} 2$ & $138(44.2)$ & $11(9.68-12.32)$ \\
\hline N3 & $64(20.5)$ & $12(10.5-13.50)$ \\
\hline $\mathrm{Nx}$ & $43(13.8)$ & $9(6.78-11.22)$ \\
\hline \multicolumn{3}{|l|}{ M stage } \\
\hline M1a & $142(45.5)$ & $13(11.56-14.44)$ \\
\hline M1b & $170(54.5)$ & $10(8.08-11.92)$ \\
\hline \multicolumn{3}{|l|}{ Effusions at diagnosis } \\
\hline MPE, Ipsilateral & $100(32.0)$ & $14(11.93-16.07)$ \\
\hline MPE, Contralateral & $2(0.6)$ & 12 (NA) \\
\hline MPE, Bilateral & $8(2.6)$ & $8(2.46-13.54)$ \\
\hline MPE, unknown & $15(4.8)$ & $9(7.28-10.73)$ \\
\hline MPCE & $9(2.9)$ & $14(12.61-15.39)$ \\
\hline MPCE + MPE & $8(2.6)$ & $9(3.39-14.62)$ \\
\hline DLN + MPE or MPCE & $10(3.2)$ & $12(9.22-14.79)$ \\
\hline $\mathrm{DM}+\mathrm{MPE}$ or MPCE & $118(37.8)$ & $10(7.96-12.05)$ \\
\hline $\mathrm{DM}+\mathrm{DLN}+\mathrm{MPE}$ or MPCE & $42(13.5)$ & $8(5.67-10.33)$ \\
\hline
\end{tabular}

Grading with the ICD-O-3 (International Classification of Diseases for Oncology) histologic grading system; NOS, not otherwise specified; T, tumor; N, node; M, metastasis; MPE, malignant pleural effusion; MPCE, malignant pericardial effusion; DLN, distant lymph nodes; DM, distant metastasis; NA, not available.

Development of the survival model. Parametric models and semiparametric models were built using the SEER training cohort. The lognormal model had an AIC of 48483.84, which was the lowest value among the Cox proportional hazard (133573.7), Weibull (49645.39), Gaussian (53229.58), exponential (50999.93), logistic (53440.71) and loglogistic (48902.85) models. The nomogram was developed based on the lognormal model (Fig. 1). For example, the effusion factor consisted of ipsilateral MPE, contralateral MPE, MPCE, effusion with distant lymph node(s) (DLN), effusion with distant metastases (DM) and effusion with both DM and DLN metastases. Ipsilateral MPE (46.0 points) had the largest point value, which predicted the best survival, whereas effusion with both DM and DLN metastasis (0 point) had the smallest point value, which predicted the worst survival rate. The scores for each variable in the nomogram are listed in Table II.

Validation and calibration of the survival model. The C-index of the survival model was 0.736 in the training cohort and 0.772 in the validation cohort. Calibration curves were drawn using the internal bootstrap method (A) and with an independent external dataset (B) in Fig. 2. These curves showed an acceptable fit between the actual and nomogram-predicted probability of OS.
Risk group stratification. The total points of the present nomogram for each patient in the training and validation cohorts were calculated. Patients in both cohorts were divided into four prognostic groups according to the quartiles of the predicted survival of the training cohort. Kaplan-Meier survival curves were constructed for all patients and for patients with certain M stages (M1a and M1b) in both the training and validation datasets. Significant differences in the 4 prognostic groups were observed for the M1a stage, M1b stage and overall dataset in both cohorts (all $\mathrm{P}<0.001$; Fig. 3).

Comparison of the nomogram with the LENT score in patients with pleural effusion. A total of 303 patients (97.1\%) in the validation dataset had pleural effusion. The median score from the LENT system for the validation dataset patients with pleural effusion was 5 (inter-quartile range 3-7). ROC curves were generated to compare the 3-, 6-, 9- and 12-month survival predictions of the LENT scoring system and the present nomogram for the patients with pleural effusion in the validation dataset. The AUC values of the present nomogram were significantly higher compared with the LENT scoring system for predicting 3-, 6- and 12-month survival (all $\mathrm{P}<0.05$; Fig. 4). The exception was the prediction of 9-month survival, for which the AUC values for the LENT score and the nomogram were 0.765 (95\% CI, 0.714-0.811) and 0.797 (95\% CI, 0.748-0.840), respectively $(\mathrm{P}=0.3513)$. 


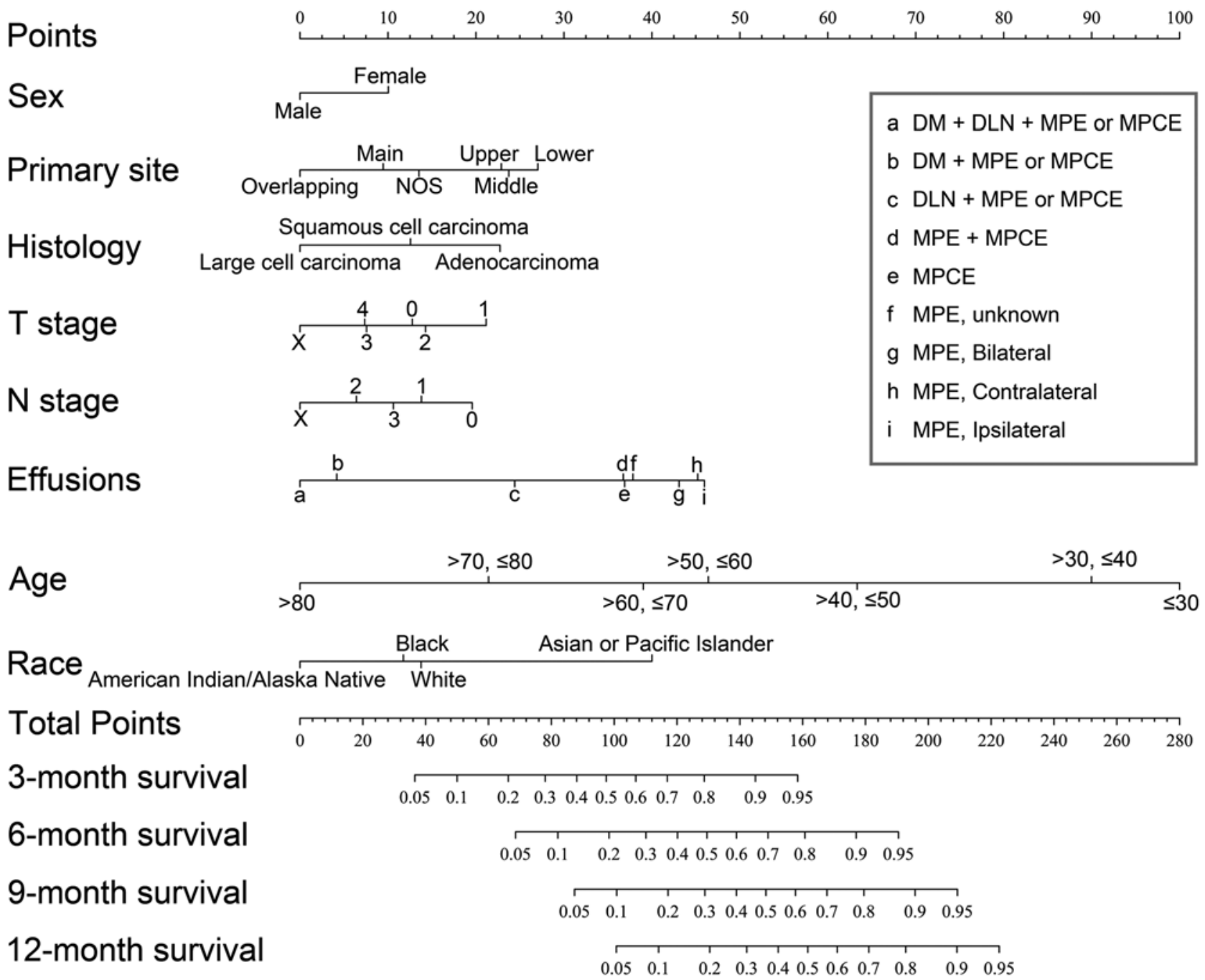

Figure 1. Prognostic nomogram for patients with NSCLC with MPE or MPCE. The points for each variable was added up to obtain the total points and the final scores were used to estimate 3-, 6-, 9- and 12-month survival. NSCLC, non-small cell lung cancer; Overlapping, overlapping lesion of lung; Main, main bronchus; NOS, not otherwise specified lesion of lung; Upper, upper lobe of lung; Middle, middle lobe of lung; Lower, lower lobe of lung; MPE, malignant pleural effusion; MPCE, malignant pericardial effusion; DLN, distant lymph node(s); DM, distant metastasis.

\section{Discussion}

MPE or MPCE is observed in $>15 \%$ of patients at initial diagnosis of NSCLC $(3,13)$. Prediction of survival is important for the stratification and management of patients because it can help physicians select appropriate therapies and identify patients who require palliative care (14). The TNM staging system allows for stratification of patients into two (M1a and M1b, AJCC 7thedition) or three (M1a, M1b and M1c, AJCC 8th edition) groups (15); however, risk group stratification showed that the present nomogram has an acceptable discrimination ability, even within a specific M stage.

Several predictive models for survival in MPE or MPCE have been published; however, a number of these are based on biomarker concentrations in the serum and/or in the effusion $(6,7,16)$. In addition to the difficulty in accessing a sufficient effusion sample in patients with a poor physical status or a low effusion volume, the biomarker concentration in a sample can vary due to different measurement techniques (17). The application of diuretics can also influence the concentration of biomarkers (18), which may limit the usability, accuracy and repeatability of those models.
A recently published study evaluated the performance of the widely used LENT scoring system with an Asian lung adenocarcinoma cohort, and reported that the LENT score underestimated the OS in this distinct group (3). This result could be due to the high prevalence of epidermal growth factor receptor (EGFR) mutations in these patients (19), and these patients may benefit from tyrosine kinase inhibitor therapy, which leads to prolonged OS (20). The nomogram developed in the present study showed that female patients (10.0 points) with adenocarcinoma (22.7 points) and Asian or Pacific Islander ethnicity (40.0 points) had the highest points for each variable, suggesting that patients with these characteristics had longer predicted survival. This may be due to the fact that patients with these characteristics have a high EGFR mutation incidence, and that EGFR mutations predict benefit from treatment $(21,22)$.

Nomograms have been shown to provide more accurate and individualized survival predictions (23). In the present study, the nomogram was accurate for the training and validation cohorts. Nomograms are also a useful tool for visualizing prognostic factors (24), and the present nomogram revealed 
Table II. Points assignment for each variable.

\begin{tabular}{|c|c|}
\hline Variables & Points \\
\hline \multicolumn{2}{|l|}{ Sex } \\
\hline Male & 0 \\
\hline Female & 10.0 \\
\hline \multicolumn{2}{|l|}{ Age at diagnosis, years } \\
\hline$\leq 30$ & 100.0 \\
\hline$>30, \leq 40$ & 90.0 \\
\hline$>40, \leq 50$ & 63.3 \\
\hline$>50, \leq 60$ & 46.4 \\
\hline$>60, \leq 70$ & 39.0 \\
\hline$>70, \leq 80$ & 21.4 \\
\hline$>80$ & 0 \\
\hline \multicolumn{2}{|l|}{ Primary site } \\
\hline Main bronchus & 9.5 \\
\hline Upper lobe & 22.9 \\
\hline Middle lobe & 23.7 \\
\hline Lower lobe & 27.1 \\
\hline Overlapping lesion & 0 \\
\hline Lung, NOS & 13.5 \\
\hline \multicolumn{2}{|l|}{ Histology } \\
\hline Large cell carcinoma & 0 \\
\hline Adenocarcinoma & 22.7 \\
\hline Squamous cell carcinoma & 12.6 \\
\hline \multicolumn{2}{|l|}{$\mathrm{T}$ stage } \\
\hline T0 & 12.8 \\
\hline $\mathrm{T} 1$ & 21.2 \\
\hline $\mathrm{T} 2$ & 14.3 \\
\hline $\mathrm{T} 3$ & 7.6 \\
\hline $\mathrm{T} 4$ & 7.4 \\
\hline $\mathrm{Tx}$ & 0 \\
\hline \multicolumn{2}{|l|}{$\mathrm{N}$ stage } \\
\hline No & 19.6 \\
\hline N1 & 13.8 \\
\hline $\mathrm{N} 2$ & 6.4 \\
\hline N3 & 10.6 \\
\hline $\mathrm{Nx}$ & 0 \\
\hline \multicolumn{2}{|l|}{ Effusions at diagnosis } \\
\hline MPE, Ipsilateral & 46.0 \\
\hline MPE, Contralateral & 45.2 \\
\hline MPE, Bilateral & 36.9 \\
\hline MPE, unknown & 43.1 \\
\hline MPCE & 37.9 \\
\hline MPCE + MPE & 24.4 \\
\hline $\mathrm{DLN}+\mathrm{MPE}$ or MPCE & 36.8 \\
\hline $\mathrm{DM}+\mathrm{MPE}$ or MPCE & 4.2 \\
\hline $\mathrm{DM}+\mathrm{DLN}+\mathrm{MPE}$ or MPCE & 0 \\
\hline
\end{tabular}

different prognoses among each of the factors. Tumor grade is an important independent prognostic marker; however, in the construction of the present prognostic model, it was found that including the tumor grade did not significantly improve
Table II. Continued.

\begin{tabular}{lc}
\hline Variables & Points \\
\hline Race & \\
American Indian/Alaska Native & 0 \\
Asian or Pacific Islander & 40.0 \\
Black & 11.7 \\
White & 13.8 \\
\hline
\end{tabular}

NOS, not otherwise specified; T, tumor; $\mathrm{N}$, node; $\mathrm{M}$, metastasis; MPE, malignant pleural effusion; MPCE, malignant pericardial effusion; DLN, distant lymph node(s); DM, distant metastasis.

the efficiency of the model (data not shown). A possible explanation for this is that the tumor grade may be interrelated with other factors in the present model and that these factors may be highly efficient. Another explanation may be that the present model focused on a specific subgroup, namely patients with stage IV NSCLC. Metastatic tumor cells may be more aggressive due to their heterogeneity, which makes the grade of primary tumor less useful for prognostic predictions (25). In the present nomogram, the value of the $\mathrm{N}$ component is not arranged regularly from small to large, as the definition of regional lymph nodes in the NSCLC TNM stage system is by lymph node location rather than by the number of positive lymph nodes, which may suggest that patients with MPE and MPCE metastases in specific lymph node locations may have a less favorable prognosis.

The AUCs of the ROC curves for the LENT scoring system in the present validation cohort were quite similar to those in the report of the LENT study (7). The AUCs were reported to be 0.7571 for 3 months and 0.8094 for 6 months in the LENT scoring system for the UK cohort 2 (7), and in the present study the AUCs were 0.812 for 3 months and 0.794 for 6 months. The ROC curves showed that the present nomogram, based on demographic and clinicopathological characteristics, may provide a similar 9-month survival prediction as that generated by the LENT scoring system and better predictions for 3-, 6- and 12-month survival. Therefore, the present nomogram may be an additional option for physicians to predict survival of patients with NSCLC with MPE or MPCE at the initial diagnosis.

The median survival of the validation cohort was longer than that of the training cohort and this may be due to differences in the races of the patients included in each cohort. However, the present nomogram still provided a good prediction of outcomes for the patients in the validation cohort, as the point values were 13.8 for white patients and 40.0 for Asian patients in the race factor of the predictive model. This observation suggested that the present nomogram remained robust in a homogeneous Asian population and thus indicated that the nomogram may be preferably used for Asian patients. Nevertheless, further studies that validate this nomogram in a cohort with races are still required.

As the SEER database contains retrospective data and uses a collaborative stage data collection system that records only coding data to protect the identities of cancer patients (26), 

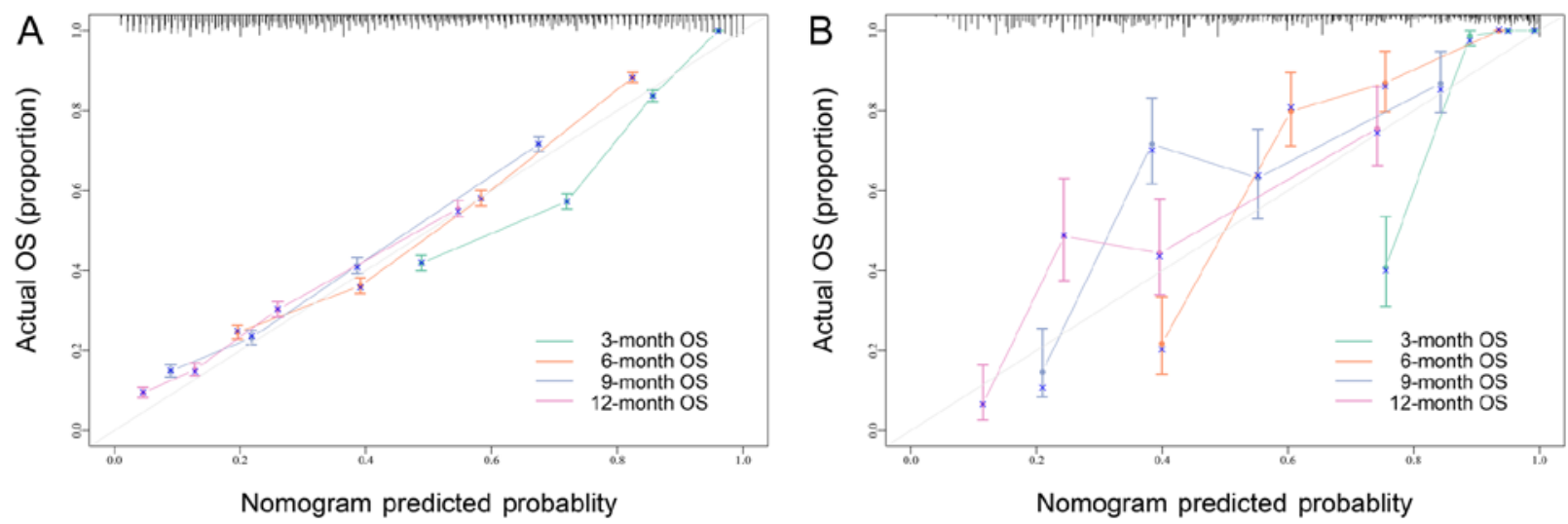

Figure 2. Calibration plots of the training and validation cohorts. The x-axis shows the proportion of actual OS, while the y-axis shows the probability. The gray reference line indicates that the nomogram predicted OS is equal to the actual OS. Data represented the means \pm standard error of the mean. (A) Internal bootstrap method; (B) External validation cohort. OS, overall survival.
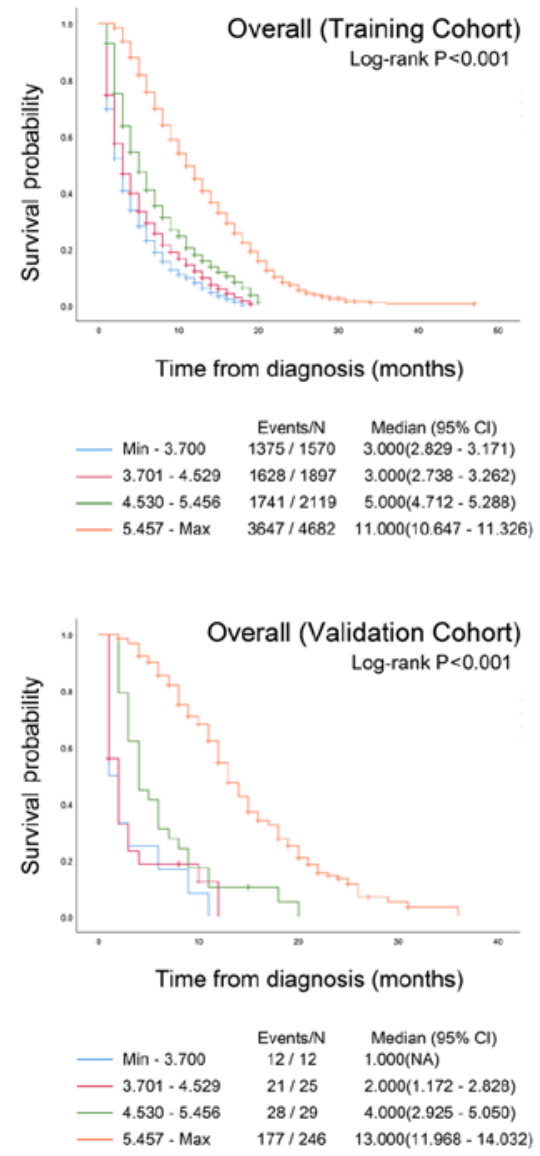

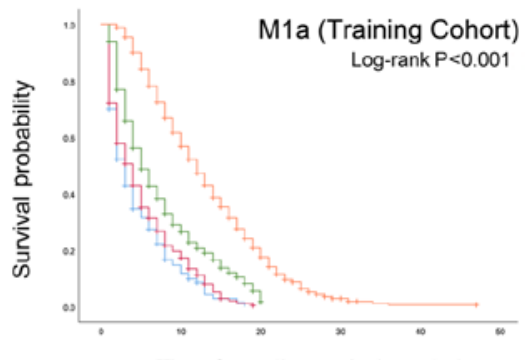

Time from diagnosis (months)
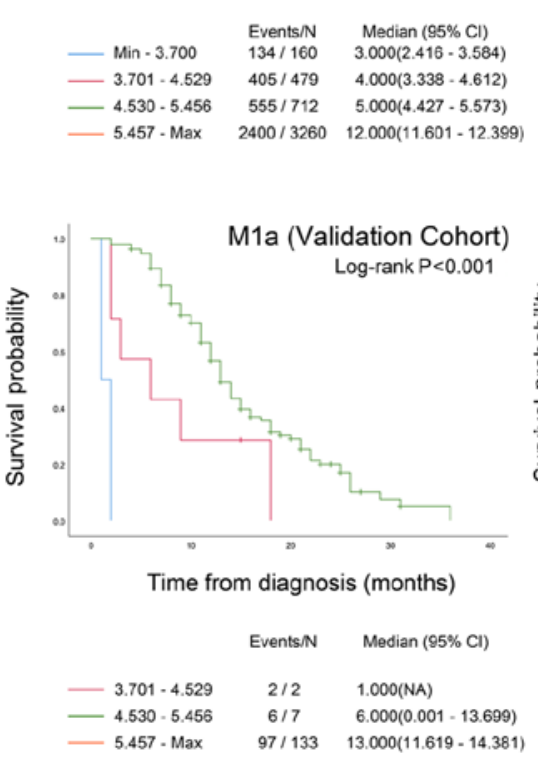
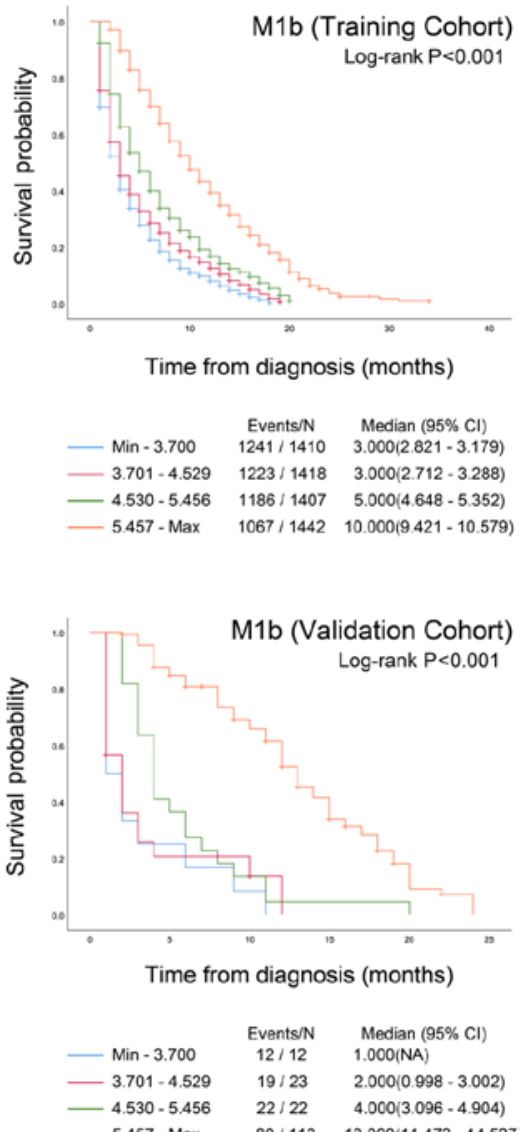

Figure 3. Risk group stratification of overall survival in the training and validation cohorts. Patients in each category were divided into four prognostic groups according to the quartiles of predicted survival. A Kaplan-Meier plot of probability of survival is presented for each quartile group. Min, Minimum; CI, confidence interval; NA, Not Applicable; Max, Maximum; Mla, according to the AJCC 7th edition of TNM staging; Mlb, according to the AJCC 7th edition of TNM staging.

it is difficult to precisely convert the patient data recorded with the AJCC 7th edition of TNM staging to the AJCC 8th edition. The nomogram of the present study is depicted with the AJCC 7th edition. However, the difference between the 7th and 8th editions is that the specific Tumor component is small and these differences are well known to specialists; however, no change was made to the Node component. Therefore, any inconsistencies caused by the differences in editions of the staging system when using the present nomogram should be minimal.

The present study had several limitations. Firstly, some of the clinicopathological variables and molecular markers, such as physical status $(27,28)$, EGFR mutation and PD-L1 status, which may influence the survival of patients with lung cancer $(22,29)$, are not included in the SEER database; however, these biomarkers are more predictive than prognostic, 
3 months

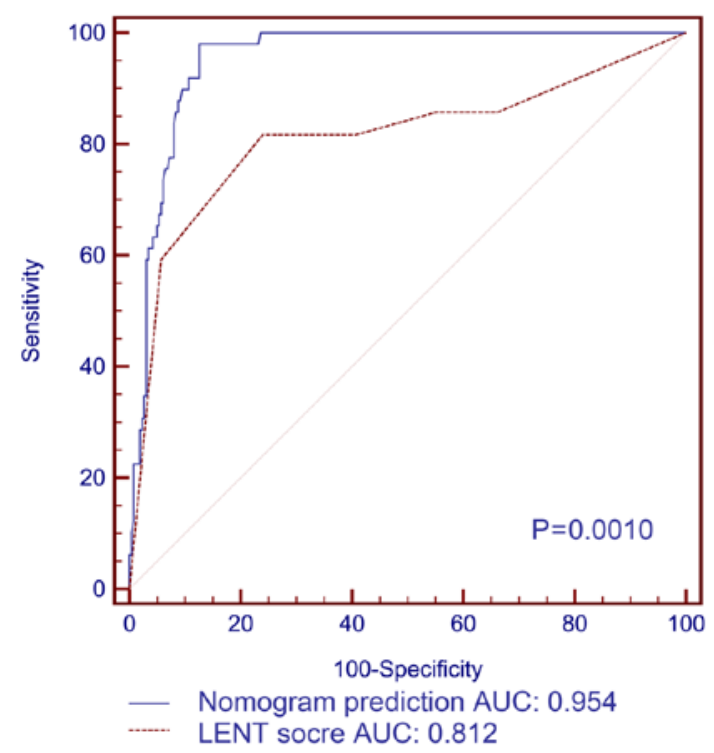

9 months

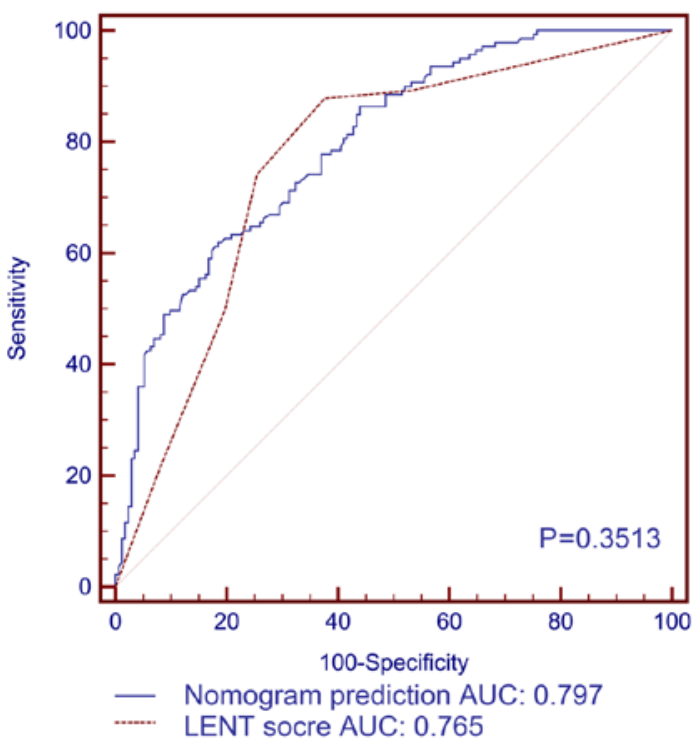

6 months

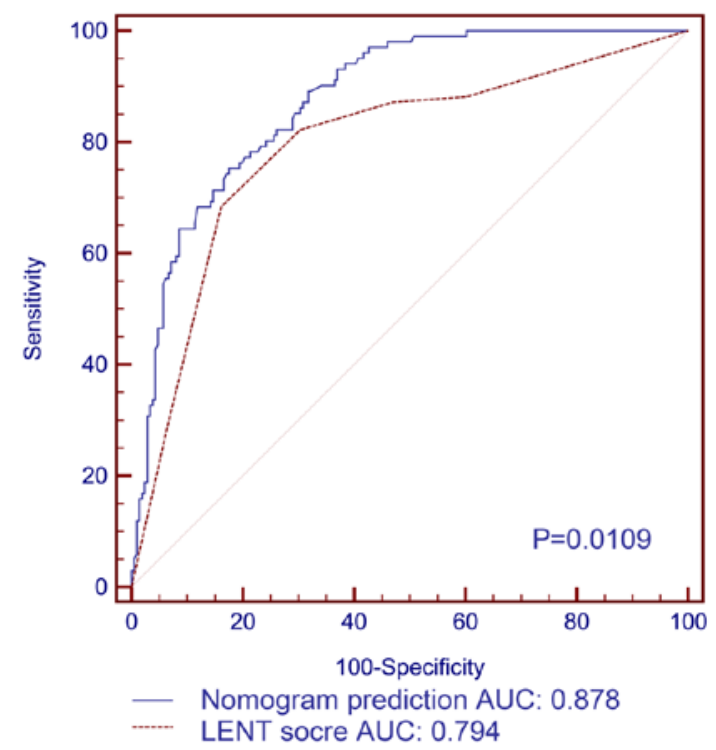

12 months

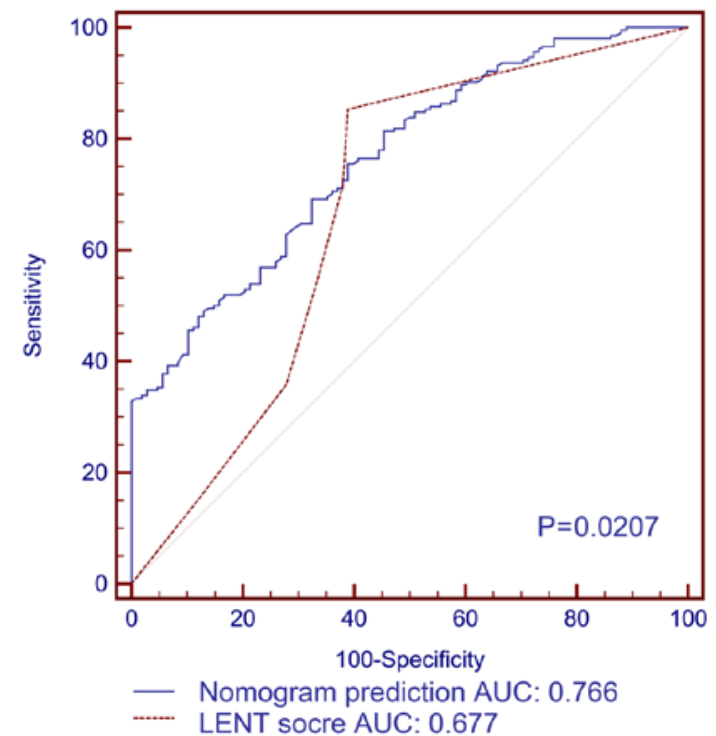

Figure 4. ROC curves of the present nomogram and the LENT scoring system in the validation cohort. ROC, receiver operating characteristic; AUC, area under the curve; LENT, the LENT (pleural fluid lactate dehydrogenase, Eastern Cooperative Oncology Group performance status, neutrophil to lymphocyte ratio and tumor type) prognostic score.

which may limit their use in a prognosis prediction model (30). Secondly, the time of disease progression was not available in the SEER database, thus it was not possible to construct predictive models of progression-free survival. Additionally, the nomogram developed in the present study performed better for cases near the median; whereas for discrete cases the predictive performance was poor, which is a common limitation of predictive models (31). MPCE was included in the present study as the SEER database used a 'CS Mets at DX' item to describe MPE or MPCE. Each patient was categorized into a specific group, such as 'DLN + MPE or MPCE', 'DM + MPE or MPCE', MPE, and 'MPE + MPCE'. Thus, it was difficult to determine whether a patient in the 'DLN + MPE or MPCE' group had MPE or MPCE, and excluding this group may have led to a bias in the predicted model. Finally, although the present nomogram was built based on a large population and was calibrated by both internal bootstrap resampling and an external cohort, the external cohort was collected from one district, which may lead to potential bias in validation and calibration, including the limited diversity of patient characteristics. A large external validation cohort is required to confirm the nomogram.

In conclusion, nomograms may provide a precise and easily understandable illustration of survival for healthcare providers of patients with lung cancer with MPE or MPCE at the initial diagnosis. In the present study, a nomogram was constructed for predicting the survival of patients with NSCLC with MPE or MPCE at initial diagnosis based on the SEER database. The nomogram developed in the present study may improve prognostic prediction and disease management for patients with lung cancer. 


\section{Acknowledgements}

Not applicable.

\section{Funding}

No funding was received.

\section{Availability of data and materials}

The datasets used and/or analyzed during the current study are available from the corresponding author on reasonable request.

\section{Authors' contributions}

TT and WH designed the study and analyzed the data. PZ, CS and JZ participated in data acquisition. FZ participated in data interpretation. All authors read and approved the final manuscript.

\section{Ethical approval and consent to participate}

The present study was approved by The Ethics Committee of Fuyang People's Hospital (Fuyang, China). Permission was obtained from the Surveillance, Epidemiology, and End Results (SEER) Program to access the SEER research data files (reference no. 16924-Nov2017). Informed consent was obtained from each patient in the validation dataset. However, consent in the SEER training cohort was waived by The Ethics Committee of Fuyang People's Hospital (Fuyang, China) considering the anonymous, observational, registry-based, and publicly available nature.

\section{Patient consent for publication}

Not applicable.

\section{Competing interests}

The authors declare that they have no competing interests.

\section{References}

1. Siegel RL, Miller KD and Jemal A: Cancer statistics, 2019. CA Cancer J Clin 69: 7-34, 2019.

2. Bray F, Ferlay J, Soerjomataram I, Siegel RL, Torre LA and Jemal A: Global cancer statistics 2018: GLOBOCAN estimates of incidence and mortality worldwide for 36 cancers in 185 countries. CA Cancer J Clin 68: 394-424, 2018.

3. Abisheganaden J, Verma A, Dagaonkar RS and Light RW: An observational study evaluating the performance of LENT score in the selected population of malignant pleural effusion from lung adenocarcinoma in Singapore. Respiration 96: 308-313, 2018.

4. Ou SH and Zell JA: Validation study of the proposed IASLC staging revisions of the T4 and $\mathrm{M}$ non-small cell lung cancer descriptors using data from 23,583 patients in the California cancer registry. J Thorac Oncol 3: 216-227, 2008.

5. Roberts ME, Neville E, Berrisford RG, Antunes G and Ali NJ; BTS Pleural Disease Guideline Group: Management of a malignant pleural effusion: British thoracic society pleural disease guideline 2010. Thorax 65 (Suppl 2): ii32-ii40, 2010.

6. Psallidas I, Kanellakis NI, Gerry S, Thézénas ML, Charles PD, Samsonova A, Schiller HB, Fischer R, Asciak R, Hallifax RJ, et al: Development and validation of response markers to predict survival and pleurodesis success in patients with malignant pleural effusion (PROMISE): A multicohort analysis. Lancet Oncol 19: 930-939, 2018
7. Clive AO, Kahan BC, Hooper CE, Bhatnagar R, Morley AJ, Zahan-Evans N, Bintcliffe OJ, Boshuizen RC, Fysh ET, Tobin CL, et al: Predicting survival in malignant pleural effusion: Development and validation of the LENT prognostic score. Thorax 69: 1098-1104, 2014.

8. Bildirici U, Celikyurt U, Acar E, Bulut O, Sahin T, Kozdag G and Ural D: The value of serum tumour markers in the prediction of aetiology and follow up of patients with pericardial effusion. Cardiovasc J Afr 23: 143-146, 2012.

9. Wang SJ, Lemieux A, Kalpathy-Cramer J, Ord CB, Walker GV, Fuller CD, Kim JS and Thomas CR Jr: Nomogram for predicting the benefit of adjuvant chemoradiotherapy for resected gallbladder cancer. J Clin Oncol 29: 4627-4632, 2011.

10. Eil R, Diggs BS, Wang SJ, Dolan JP, Hunter JG and Thomas CR: Nomogram for predicting the benefit of neoadjuvant chemoradiotherapy for patients with esophageal cancer: A SEER-Medicare analysis. Cancer 120: 492-498, 2014.

11. Vergouwe Y, Steyerberg EW, Eijkemans MJ and Habbema JD: Substantial effective sample sizes were required for external validation studies of predictive logistic regression models. J Clin Epidemiol 58: 475-483, 2005.

12. DeLong ER, DeLong DM and Clarke-Pearson DL: Comparing the areas under two or more correlated receiver operating characteristic curves: A nonparametric approach. Biometrics 44: $837-845,1988$.

13. Porcel JM, Gasol A, Bielsa S, Civit C, Light RW and Salud A: Clinical features and survival of lung cancer patients with pleural effusions. Respirology 20: 654-659, 2015.

14. Mbeutcha A, Mathieu R, Rouprêt M, Gust KM, Briganti A, Karakiewicz PI and Shariat SF: Predictive models and prognostic factors for upper tract urothelial carcinoma: A comprehensive review of the literature. Transl Androl Urol 5: 720-734, 2016

15. Yang L, Wang S, Zhou Y, Lai S, Xiao G, Gazdar A and Xie Y: Evaluation of the 7th and 8th editions of the AJCC/UICC TNM staging systems for lung cancer in a large North American cohort. Oncotarget 8: 66784-66795, 2017.

16. Tian T, Li J, Hu W, Sun C and Zhou J: Thymidine kinase 1 concentration in pleural effusion is a diagnostic marker and survival predictor for malignant pleural effusion. J Clin Lab Anal 33: e22901, 2019.

17. Psallidas I, Kalomenidis I, Porcel JM, Robinson BW and Stathopoulos GT: Malignant pleural effusion: From bench to bedside. Eur Respir Rev 25: 189-198, 2016.

18. Liu YY: Influencing factors and combined application of chemiluminescence immunoassay for detection of tumor markers. Clin Lab J 1: 143, 2019.

19. Ren S, Kuang P, Zheng L, Su C, Li J, Li B, Chen X, Wang Y, KimCurran V, Liu L, et al: Analysis of driver mutations in female non-smoker Asian patients with pulmonary adenocarcinoma. Cell Biochem Biophys 64: 155-160, 2012.

20. Thatcher N, Chang A, Parikh P, Rodrigues Pereira J, Ciuleanu T, von Pawel J, Thongprasert S, Tan EH, Pemberton K, Archer V and Carroll K: Gefitinib plus best supportive care in previously treated patients with refractory advanced non-small-cell lung cancer: Results from a randomised, placebo-controlled, multicentre study (Iressa Survival Evaluation in Lung Cancer). Lancet 366: 1527-1537, 2005.

21. Midha A, Dearden S and McCormack R: EGFR mutation incidence in non-small-cell lung cancer of adenocarcinoma histology: A systematic review and global map by ethnicity (mutMapII). Am J Cancer Res 5: 2892-2911, 2015.

22. Zhou Q, Zhang XC, Chen ZH, Yin XL, Yang JJ, Xu CR, Yan HH, Chen HJ, Su J, Zhong WZ, et al: Relative abundance of EGFR mutations predicts benefit from gefitinib treatment for advanced non-small-cell lung cancer. J Clin Oncol 29: 3316-3321, 2011.

23. Iasonos A, Schrag D, Raj GV and Panageas KS: How to build and interpret a nomogram for cancer prognosis. J Clin Oncol 26: 1364-1370, 2008.

24. Van Belle V and Van Calster B: Visualizing risk prediction models. PLoS One 10: e0132614, 2015.

25. Du F: Differentiation antigen expression and lung cancer heterogeneity. J Clin Exp Pathol 2: 200-203, 2002.

26. Chen VW, Ruiz BA, Hsieh MC, Wu XC, Ries LA and Lewis DR: Analysis of stage and clinical/prognostic factors for lung cancer from SEER registries: AJCC staging and collaborative stage data collection system. Cancer 120 (Suppl 23): 3781-3792, 2014.

27. Dixit R, Agarwal KC, Gokhroo A, Patil CB, Meena M, Shah NS and Arora P: Diagnosis and management options in malignant pleural effusions. Lung India 34: 160-166, 2017. 
28. Zamboni MM, da Silva CT Jr, Baretta R, Cunha ET and Cardoso GP: Important prognostic factors for survival in patients with malignant pleural effusion. BMC Pulm Med 15: 29, 2015.

29. Garon EB, Rizvi NA, Hui R, Leighl N, Balmanoukian AS Eder JP, Patnaik A, Aggarwal C, Gubens M, Horn L, et al: Pembrolizumab for the treatment of non-small-cell lung cancer. N Engl J Med 372: 2018-2028, 2015

30. Ballman KV: Biomarker: Predictive or prognostic? J Clin Oncol 33: 3968-3971, 2015.
31. Vickers AJ and Cronin AM: Everything you always wanted to know about evaluating prediction models (but were too afraid to ask). Urology 76: 1298-1301, 2010

(i) (2) This work is licensed under a Creative Commons

cc) $\mathrm{EY}$ NC No Attribution-NonCommercial-NoDerivatives 4.0 International (CC BY-NC-ND 4.0) License. 